\title{
EVALUATION OF RESULTS OF ARTHROSCOPIC BANKART REPAIR FOR POST TRAUMATIC ANTERIOR SHOULDER INSTABILITY
}

Mainak Chandra1 ${ }^{1}$, Subhadeep Basak², Debasish Bhar³, Piyali Mondal', Partha Sarathi Halder, Anjan Das ${ }^{6}$

\section{HOW TO CITE THIS ARTICLE:}

Mainak Chandra, Subhadeep Basak, Debasish Bhar, Piyali Mondal, Partha Sarathi Halder, Anjan Das. "Evaluation of Results of Arthroscopic Bankart Repair for Post Traumatic Anterior Shoulder Instability".Journal of Evolution of Medical and Dental Sciences 2015; Vol. 4, Issue 66, August 17; Page: 11459-11467,

DOI: $10.14260 /$ jemds/2015/1654

ABSTRACT: BACKGROUND: The study was to evaluate the surgical outcomes of arthroscopic repair of post-traumatic Bankart lesions with the use of suture anchors. Patients with $>20 \%$ bony lesions, SLAP (superior labral tear from anterior to posterior) lesions and multi-directional instability were excluded. The patients were followed up for a period of minimum 4 years. MATERIALS AND METHODS: We evaluated the results of arthroscopic Bankart repair with use of suture anchors in 35 patients with traumatic recurrent anterior instability of the shoulder. The mean age at operation was 25.71 years. The patients were evaluated pre-operatively and at follow-up using the UCLA (University of California Los Angeles) shoulder scoring system and the modified Rowe scores, which were 6.2 and 29.3 respectively pre-operatively. RESULTS: The UCLA shoulder scoring system and the modified Rowe scores at follow-up were 32 and 72.57 respectively and both improvements were significant. The Modified Rowe Shoulder Scoring System showed 14 patients having excellent results, 12 patients good, 6 patients fair and 3 patients with poor results. One patient had subluxation and another had positive apprehension test. Five patients had discomfort/pain with arm in abducted and externally rotated position but negative apprehension test. Remaining 28 patients had negative apprehension test; no subluxation. Significant improvements occurred for each motion tested for each follow up visit. CONCLUSION: We conclude that arthroscopic Bankart lesion repair with suture anchors is an effective surgical technique for the treatment of an isolated Bankart lesion having good results with respect to pain relief, stability and function.

KEYWORDS: Arthroscopic Bankart repair, Anterior shoulder instability, UCLA (University of California Los Angeles) shoulder rating scale.

INTRODUCTION: The shoulder, by virtue of its anatomy and biomechanics, is one of the most unstable and frequently dislocated joints in the body, accounting for nearly $50 \%$ of all dislocations. According to one estimate, up to $96 \%$ of acute shoulder dislocations were traumatic in origin.(1)

Anterior instability is the most common form of shoulder instability.(2) Recurrent anterior shoulder instability results in a functional disability for the patient, in terms of both shoulder function and general health status.(3)

The use of arthroscopy has improved the recognition of pathologic findings in shoulder instability and allowed a better understanding of the etiology of instability and the correlation between symptom and lesion patterns.

Arthroscopic treatment of shoulder instability due to Bankart lesion introduced some advantages compared with open Bankart lesion repair procedure. The Arthroscopic method offers a less invasive technique of Bankart repair. 
Arthroscopic shoulder stabilization utilizing the arthroscopic instrumentation system has particular application for post-traumatic anterior instability.

The goal is to reattach the capsuloligamentous complex under sufficient tension and anatomic approximation to facilitate normal shoulder translation while retaining a normal range of motion. Patients with a detached labrum and capsule with normal glenohumeral ligaments are preferred.(4) This study aims to evaluate the outcome of arthroscopic Bankart repair with the use of suture anchors for cases that were followed-up for at least 4 years from the date of surgery.

MATERIALS AND METHODS: After obtaining permission from institutional ethics committee, written informed consent was taken from all 40 adult patients for this interventional, longitudinal (Prospective) study.

\section{Inclusion Criteria:}

1. Patients with Post traumatic Bankart lesion as detected per-operatively by arthroscopy.

2. Absence of other capsular and tendon injuries.

3. Minimal bony lesion $(<20 \%)$-assessed per-operatively by arthroscopy using a probe.

4. No clinical evidence of multidirectional instability.

\section{Exclusion Criteria:}

1. Bankart lesion with SLAP lesion.

2. Generalized ligamentous laxity.

3. Large bony lesion (>20\%)-assessed per-operatively by arthroscopy.

4. Associated relevant co-morbid features e.g. Seizure disorder.

\section{Parameters to be Studied:}

1. Independent Variables- Age, Sex, Type of Injury, Number of Recurrent dislocations, Side of dislocation, Time gap between $1^{\text {st }}$ Dislocation- surgical interventions.

2. Dependent Variables- Pain, Movement, Function, Stability, Patient satisfaction.

3. Any risk factor(s) for failure.

The anchors used were Poly Lactic Acid Absorbable Anchor \& titanium non-absorbable Anchors. The patients were evaluated during follow up using the UCLA Shoulder rating scale and the Modified Rowe scale.

Pre-operative Planning: Patients were evaluated on Clinico-radiological basis. MRI findings of some patients did not correlate well with arthroscopic findings. Clinical- Tests for instability \& ROM.

Radiological- X-ray of affected shoulder-AP view (to exclude OA changes \& associated fractures) and MRI of affected shoulder were done. Pre-operative modified Rowe score was 29.3 and the preoperative mean UCLA functional score was 6.2.

Operative Procedures: Patient is positioned on lateral decubitus position after achieving proper regional anaesthesia (Usually supra-scapular block with inter-scalene brachial plexus anaesthesia). Stability testing was performed under anesthesia to confirm the clinical diagnosis before arthroscopy. A standard posterior arthroscopic portal was established. The arthroscope was placed through the standard posterior portal and used to create two working portals anteriorly. 


\section{ORIGINAL ARTICLE}

Identification of Lesion: A diagnostic arthroscopy was performed, and the presence of the Bankart lesion (An anterior inferior avulsion of the capsulolabral complex of the glenoid was confirmed. SLAP lesion, rotator cuff injuries, biceps tendon injuries and any engaging Hill Sach's lesion were ruled.

Glenoid Preparation: A Knife Rasp was first used to dissect the capsule and labrum off the neck of the glenoid. Shaver and rasp were then used to further roughen the glenoid neck to bleeding bone.

Suture Anchor Placement: Two or three suture anchors were placed between the 3o'clock and 5 o'clock positions. It was imperative to place the suture anchor on the apex of the glenoid rim to allow restoration of concentricity and compression. Proper seating of the suture anchor was checked by placing tension on the sutures.

Suture Passing Technique: The patient's arm was placed in $30^{\circ}$ abduction in preparation for passing sutures. Next, the clever hook was passed through the inferior glenohumeral ligament (IGHL) and used to retrieve the free suture end and withdraw it out of the cannula.

Knot Tying Procedure: The free ends of suture were tied with a three and half-hitch knot (Fisherman knot) and advanced down the cannula with the Shoulder Knot Pusher. These anchors are used to relocate, hold in place and tighten the injured IGHL.

Assessment of Suture Placement: A probe through the anterior portal was used to ensure firm opposition of capsule to glenoid rim. Next, the arthroscope was passed through the anterior portal to visualize the anterior glenoid and suture placement.

RESULTS: 40 Patients, each with unilateral Bankart lesion [Fig-1], were initially taken up for the study. 5 patients were lost to follow up. The study was conducted on remaining 35 patients for minimum follow up period of 4 years (range 48 to 54 months).

Intra-operative Findings: All thirty five patients selected for this study were right handed. Twenty three patients had extension of labral injury ranged from 6 o'clock to 1 o'clock (affecting right shoulder).Twelve patients had extension of labral injury range from 10 o'clock to 6 o' clock position (Affecting left shoulder) [Table-1] [Fig-1]. All patients were treated [Fig-1] with 2(71.4\%) or 3 (28.6\%) anchors [Table-2]. Thermal capsulorrhaphy was not used. The lateral decubitus position was used for all thirty five patients in our study.

Follow up Findings: We did not see any intraoperative or postoperative complications. One patient had subluxation (Who was a male athlete with overhead throwing activities) and another had positive apprehension test (Among the general population). Five patients had discomfort or pain with arm in abducted and externally rotated position but negative apprehension test. Remaining 28 patients had negative apprehension test; no subluxation. Significant improvements occurred for each motion tested for each follow up visit. Strength was measured on a 5-point MRC grading scale. At the follow-up visit, $80 \%$ of patients had strength rating of 5 of 5 in forward flexion, $20 \%$ of patients had strength rating of 4 of 5 in forward flexion [Table-3]. 
Subjective Assessments: All patients demonstrated significant improvements in the UCLA shoulder scoring system and Modified Rowe Shoulder Scoring Systems at follow-up. The mean UCLA score was 32 of 35 points, with $80 \%$ good to excellent results [Table-4]. The Modified Rowe Shoulder Scoring System improved to 75.3 with 14 patients having excellent results, 12 patients good, 6 patients fair and 3 patients with poor results [Table-5].

DISCUSSION: Recurrent anterior shoulder instability results in a functional disability for the patient in terms of both shoulder function and general health status.(2) The shoulder, by virtue of its anatomy and biomechanics, is one of the most unstable and frequently dislocated joints in the body, accounting for nearly $50 \%$ of all dislocations. As per different literatures, the reported incidence being 8.2 to 23.9 per 100,000 persons per year.(5) In most patients however, shoulder instability occurs after a clear traumatic insult. According to one estimate, up to $96 \%$ of acute shoulder dislocations were traumatic in origin.(1)

In our study, shoulder instability occurred following traumatic insult in all patients with mean age of 25.71 years (17-38 yrs.). Our study population included 35 patients with 32 men (91.43\%) and 3 women (8.57\%). Mean number of episodes of dislocation were 5.85 (2-15). In our study mean number of dislocation were more than other standard studies, probably due to majority of our study population belonging to low socio-economic status and lack of awareness.

At the end of 4 years follow up, there was one patient with positive apprehension test and one with sense of subluxation; 5 patients presented with discomfort or pain with arm in abducted and externally rotated position but with negative apprehension test. We have used the UCLA score for evaluating the results in our series with $80 \%$ good to excellent results. The Modified Rowe Shoulder Scoring System improved to 75.3 with 14 patients having excellent results, 12 patients good, 6 patients fair and 3 patients with poor results.

In the study of Carreira et al.(6) 85 patients including 70 men (82.4\%) and 15 women with mean age 24.5 years (19-33 yrs) were chosen for arthroscopic Bankart repair. 10\% patients experienced recurrent instability after repair with minimum of 2 years follow-up. They evaluated their results using Rowe scores with $90 \%$ good to excellent results. 29 patients required 2 anchors, 41 patients requiring 3 anchors. The number of anchors used averaged 2.58.Bioabsorbable anchor $(3.5 \mathrm{~mm})$ was used.

Cho et al.(7) chosen 62 patients including 55 men (88.7\%) and 7 women $(11.3 \%)$ for arthroscopic Bankart repair. No recurrent dislocation or anterior instability during physical examination was noted with minimum of 1 year follow up. They evaluated their results using American Shoulder and Elbow Surgeon (ASES) scoring system with 85\% good or excellent results.

Fabbriciani et al.(8) Recruited 60 patients including 50 men (83\%) and 10 women (17\%) with their mean age 26.8 yrs. (21-30 yrs) for arthroscopic Bankart repair. 8\% patients experienced recurrent instability after repair with minimum of 2 years follow-up. They evaluated their results using Rowe shoulder scores with $83 \%$ good to excellent results, mean values were (Function=45.5, pain=8.5, stability=27, ROM=10,total=91).

In the study of Cho et al.(7) 62 patients with recurrent anterior shoulder dislocation due to Bankart lesion that underwent arthroscopic repair with mean age at surgery was 24.2 years (16-38 yrs). 
In the study of Fabbriciani et al.(8) mean number of episodes of dislocation were 3.2.(2-4) In the study of Carreira et al.(6) mean number of episodes of dislocation were 3.5.(2-6) In the study of Cho et al. mean no. of episodes of shoulder dislocation were 3.7.(2-6)

Mean time from first dislocation to surgery in study of Fabbriciani et al.(8) was 25.3 months (8-52 months), in the study of Carreira et al.(6) was 27.2 months (10-61 months). In our study, mean time from first dislocation to surgery was 55.4 months (12-180 months); this increase interval probably due to majority of our study population belonged to low socio-economic status and lack of awareness.

In the study of Fabbriciani et al,(8) Cho et al the patients were placed in the beach-chair position with the index arm in a pneumatic arm holder. In the study of Carrira et al.(6) the patient was placed in the lateral decubitus position. In our study we have used lateral decubitus position.

In our study, we have used 3 anchors for 10 patients, 2 anchors for 25 patients (3.5 mm PLA absorbable anchor with Orthocord were used). The number of anchors used averaged 2.28. Fabbriciani et al. (8) used 3 suture anchors. Bioabsorbable anchor ( $3.5 \mathrm{~mm})$ was used.

In the study of Cho et al.(7) they used an absorbable Panaloc (Mitek, Norwood, MA, USA) anchors $(3.5 \mathrm{~mm})$.Suture loop shuttle technique using a No. 2 Nylon suture was performed to prevent the suture strand from being twisted and the Samsung Medical Centre knot was made.

In study of Fabbriciani et al.(8) the repair of the glenoid labrum was performed according to the SCOI technique described by Snyder et al,(9) and using 3 metal threaded suture anchors $(3.5 \mathrm{~mm}$ mini-Revo; Linvac, Largo, FL) loaded with a No. 1 braided nonabsorbable suture.

In the study of Loutzenheiser et al.(10) have shown that the 2 knots used in their study have the "highest knot-holding capacity when compared with other knot configurations for braided suture". They also demonstrated "no significant differences in holding capacity between configurations tied by hand compared with those tied with a knot pusher."

In the study of Cho et al.(7) Samsung Medical Center knot was made.

In the study of Carreira et al.(6) a sliding Duncan loop knot was used for suture fixation. However if they faced any difficulty during sliding the knot, they employed a reversed-direction, switched-post, multiple-half-hitch revo knot.

In our study Fisherman's knot with multiple-half-hitch was given.

In the study of Cho et al.(7) follow-up results according to Modified Rowe Score at 2 yrs., mean values were(function=43.2, pain=8.5, stability $=28.1, \mathrm{ROM}=9.1$, Total=88.5).

The limitations of our study include the very few female patients, inadequate population of general patients and athletes to conclude anything about the causative factors for recurrence.

To conclude, Arthroscopic Bankart lesion repair with suture anchors is an effective surgical technique for the treatment of an isolated Bankart lesion. The results of arthroscopic Bankart repair for patients with recurrent traumatic anterior shoulder dislocation were good and appreciated in pain relief, stability and function according to UCLA score and Modified Rowe score. The results of this Arthroscopic technique compared favorably with those of previously reported Arthroscopic Bankart repairs. 


\section{ORIGINAL ARTICLE}

\section{RESULTS:}

\begin{tabular}{|c|c|}
\hline Variables & Numbers of Patients \\
\hline Age (in years) & 5 \\
$<20$ & 10 \\
$20-24$ & 11 \\
$25-29$ & 6 \\
$30-34$ & 3 \\
$35-40$ & $25.71(17-38)$ \\
\hline Mean Age (in years) & $32(91.4 \%): 3(8.6 \%)$ \\
\hline Gender & \\
Male: Female & $23(65.7 \%): 12(34.3 \%)$ \\
\hline Affected Side & $5.85(2-15)$ \\
\hline Dominant (Right): Non Dominant (Left) & $22.7(16-28)$ \\
\hline Episodes of Dislocation (no) & $55.4(12-180)$ \\
\hline Age of First Dislocation (in yrs) & $2(5.7 \%)$ \\
\hline Interval bet.1st Dislocation to Operation(in months) & $5(14.3 \%)$ \\
\hline Athletes with overhead throwing activities & $28(80 \%)$ \\
Athletes without overhead throwing activities & \\
Non-athletes & \\
\hline Table 1: Comparison of Demographic Data within the Study Group
\end{tabular}

\begin{tabular}{|c|c|}
\hline Variables & No. of shoulder cases (35) \\
\hline Operative Findings & 30 \\
Isolated Bankart lesion & 3 \\
Bankart lesion with Hill-Sach's lesion (mild grade) & 2 \\
Bony Bankart lesion <20\%(bone fragment was left in situ) & $25(71 \%)$ \\
\hline No. of anchors used & $10(29 \%)$ \\
2(Two) & 3(Three) \\
\hline Table 2: Comparison of Operative Findings within the Study Groups
\end{tabular}

\begin{tabular}{|c|c|c|c|c|}
\hline $\begin{array}{c}\text { Post- } \\
\text { operative } \\
\text { months }\end{array}$ & $\begin{array}{c}\text { Average pain } \\
\text { score } \\
\text { (1-unbearable } \\
\text { pain) } \\
\text { (10- no pain) }\end{array}$ & $\begin{array}{c}\text { UCLA } \\
\text { functional } \\
\text { score } \\
\text { (1-unable to } \\
\text { use limb) } \\
\text { (10-normal } \\
\text { activities) }\end{array}$ & $\begin{array}{c}\text { Average active } \\
\text { forward flexion } \\
\text { score } \\
\text { (max score 5) }\end{array}$ & $\begin{array}{c}\text { Strength of forward flexion } \\
\text { (manual muscle testing) } \\
\text { (max score 5) }\end{array}$ \\
\hline 6 & 3.028 & 2.31 & 2.51 & 3.02 \\
\hline 12 & 3.35 & 3.14 & 3.171 & 3.08 \\
\hline 18 & 3.628 & 3.75 & 3.35 & 3.54 \\
\hline 24 & 5.33 & 4.34 & 3.54 & 3.75 \\
\hline
\end{tabular}


ORIGINAL ARTICLE

\begin{tabular}{|l|c|c|c|c|}
\hline 30 & 6.51 & 5.1 & 3.95 & 3.89 \\
\hline 36 & 8.01 & 7.51 & 4.14 & 4.23 \\
\hline 42 & 8.41 & 8.59 & 4.48 & 4.5 \\
\hline 48 & 8.86 & 9.1 & 4.62 & 4.8 \\
\hline \multicolumn{7}{|r|}{ Table 3: Evaluation of Patients as per UCLA Shoulder Scoring System }
\end{tabular}

\begin{tabular}{|c|c|}
\hline Follow up Month & Average UCLA Score \\
\hline $\mathbf{6}$ & 11.82 \\
\hline $\mathbf{1 2}$ & 13.485 \\
\hline $\mathbf{1 8}$ & 15.55 \\
\hline $\mathbf{2 4}$ & 18.91 \\
\hline $\mathbf{3 0}$ & 24.33 \\
\hline $\mathbf{3 6}$ & 28.37 \\
\hline $\mathbf{4 2}$ & 30.11 \\
\hline $\mathbf{4 8}$ & 32 \\
\hline
\end{tabular}

Table 4: Mean UCLA score during follow up period

Excellent /Good (score>=27) 80\%; Fair /Poor (score<27) 20\%

\begin{tabular}{|c|c|}
\hline Age (in years) & Mean Score \\
$<20$ & 81 \\
$20-24$ & 80 \\
$25-29$ & 70.9 \\
$30-34$ & 75.83 \\
$35-40$ & 65 \\
\hline Results (\% of Patients) & Score \\
Excellent (40) & $90-100$ \\
Good (34.3) & $70-89$ \\
Fair (17.1) & $40-69$ \\
Poor (8.6) & $<40$ \\
\hline Gender & $\mathbf{7 7 . 3 : 5 3 . 8}$ \\
Male: Female & $\mathbf{7 3 . 9 :} \mathbf{7 0}$ \\
\hline Affected Side & Dominant: Non Dominant \\
Table 5: Modified Rowe score at 4 year Follow up
\end{tabular}




\section{ORIGINAL ARTICLE}

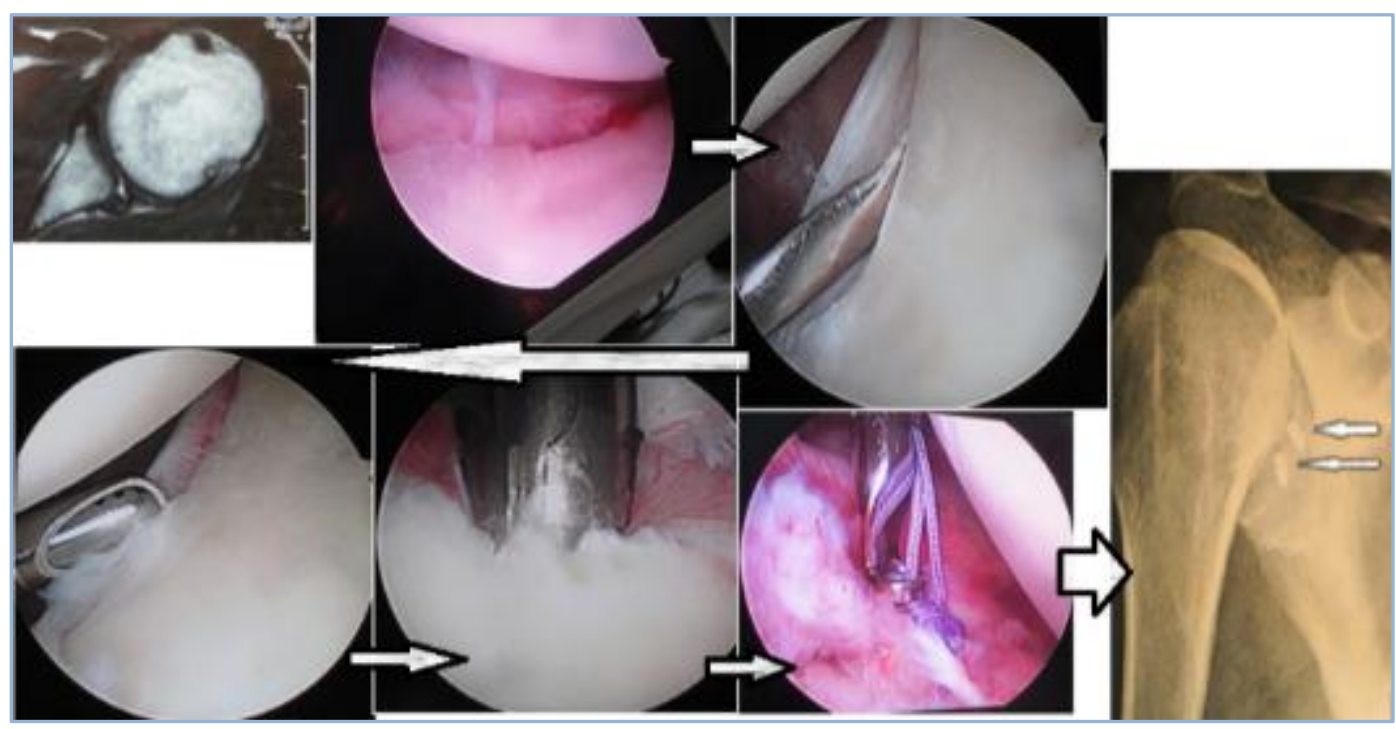

Figure 1: Steps of Arthroscopic Shoulder Surgery

\section{REFERENCES:}

1. Nordqvist A, Petersson CJ. Incidence and causes of shoulder girdle injuries in an urban population. J Shoulder Elbow Surg 1995; 4: 107-112.

2. O'Brein SJ, Neves MC, Arnoczky SP, Rozbruck SR, Dicarlo EF, Warren RF et al. The anatomy histology of the inferior glenohumeral ligament complex of the shoulder. Am J Spors Med 1990; 18: 449-456.

3. Kirkley A, Griffin S, Richards C, Mohtadi N, Miniaci A. Prospective randomized clinical trial comparing the effectiveness of immediate arthroscopic stabilization versus immobilization and rehabilitation in first traumatic anterior dislocations of the shoulder. Arthroscopy 1999; 15: 507-514.

4. Itoi E, Motzkin N, Morrey BF, An KN. Scapular inclination and inferior stability of the shoulder. J Shoulder Elbow Surg 1992; 1: 131-139.

5. Kazar B, Relovszky E. Prognosis of primary dislocation of the shoulder. Acta Orthop Scand 1969; 40: 216-224.

6. Carreira DS, Mazzocca AD, Oryhon J, Brown FM, Hayden JK, Romeo AA. A prospective outcome evaluation of arthroscopic Bankart Repairs Minimum 2-yr follow-up. Am J Sports Med 2006; 34; 771-77.

7. Cho HL, Lee CK, Hwang TH, Suh KT, Park JW. Arthroscopic repair of combined Bankart and SLAP Lesions: Operative techniques and clinical results. Clin Orthop Surg 2010; 2: 39-46.

8. Fabbriciani C, Milano G, Demontis A, Fadda S, Ziranu F, Mulas PD. Arthroscopic versus open treatment of Bankart lesion of the shoulder: A prospective randomized study. Arthroscopy 2004; 20: 456-462.

9. Snyder SJ, Karzel RP, Del Pizzo W, Ferkel RD, Friedman MJ. SLAP lesions of the shoulder. Arthoscopy 1990; 6: 274-9.

10. Loutzenheiser TD, Harryman DT, Yung SW, France MP, Sidies JA. Optimizing arthroscopic knots. Arthroscopy 1995; 11: 199-206. 


\section{ORIGINAL ARTICLE}

\section{AUTHORS:}

1. Mainak Chandra

2. Subhadeep Basak

3. Debasish Bhar

4. Piyali Mondal

5. Partha Sarathi Halder

6. Anjan Das

\section{PARTICULARS OF CONTRIBUTORS:}

1. Assistant Professor, Department of Orthopaedics, Malda Medical College, Malda, W. B.

2. Assistant Professor, Department of Obstetrics and Gynaecology, College of Med and Sagore Dutta Hospital, Kolkata.

3. Assistant Professor, Department of Anaesthesiology, Midnapore Medical College and Hospital, WB.

FINANCIAL OR OTHER

COMPETING INTERESTS: None
4. Junior Resident, Department of Physical Medicine and Rehabilitation, Sambhunath Pandit Hospital, Kolkata.

5. Associate Professor, Department of Anaesthesiology, College of Med and Sagore Dutta Hospital, Kolkata.

6. Assistant Professor, Department of Anaesthesiology, College of Med and Sagore Dutta Hospital, Kolkata.

\section{NAME ADDRESS EMAIL ID OF THE CORRESPONDING AUTHOR:}

Dr. Anjan Das, 174, Gorakshabashi Road,

Royal Plaza Apartment, $4^{\text {th }}$ Floor, Flat No.1, Nagerbazar, Dumdum.

E-mail: anjan2k8@yahoo.com

Date of Submission: 23/07/2015.

Date of Peer Review: 24/07/2015.

Date of Acceptance: 10/08/2015.

Date of Publishing: 14/08/2015. 